\title{
On the Structure of Cold Vortex
}

\author{
by \\ T. Sato \\ Meteorological Research Institute
}

(Received September 20, 1951)

\begin{abstract}
Here was derived theoretically the three-dimensional structure of the cold vortex satisfying the following conditions :

(A) the effect of friction can be considered to be secondary,

(B) the state is axis-symmetrical,

(C) the state is stationary,

(D) the air is autobarotropic.

The integrals derived under the above conditions were three, which express respectively the law of conservation of angular momentum, vorticity and energy.

From the last law can be determined the shape of the surfaces of discontinuity which can exist in a cold vortex. One of them is a surface of cold front type which stretches upward in a funnel shape sustaining a cold core in the troposphere, and the other the tropopause funnel which hangs down also in a funnel shapa surrounding the upper warm core.

In the cold vortex there exist, thus, a cold core in the lower layer containing the central axis, and a warm core which is generated by the descended stratospheric air in the upper layer. Surrounding the cores, which are rotating slowly and anticyclonically around the axis, there exists outside the funnel-shaped surfaces a tropospheric air rotating cyclonically with a large speed and containing in itself a meridional convective circulation.

As, however, the effect of friction is especially large at the surface of discontinuity, so that the outer cyclonic horizontal circulation reaches mostly to the central axis, and thus a highlevel cyclone is formed, which is represented approximately by RANKINE's combined vortex.
\end{abstract}


The three-dimensional structure of the cold vortex thus derived will be known to be remarkably similar to that of the typhoon and that of the circumpolar vortex which we described before. It would be natural that they resemble each other, because they are all phenomena which can be explained from the same fundamental principles.

\section{Integration of the equations of motion and the meaning of the derived integrals}

In the previous paper [1] it was stated that a tropopause funnel hangs down in the central part of a typhoon, and that there exists in the funnel a warm core generated by descended stratospheric air and reaching down to the earth's surface. Therein it was stated further that there can also exist a cold core under some conditions in the central part of an axis-symmetrical perturbation system.

In the cold vortex (Kaltluftropfen [2]), a cold air mass is isolated in the lower part of the atmosphere near the ground, and the surface of discontinuity bordering it stretches upward in a funnel shape in the inverse way to the case of tropopause funnel. Surrounding the funnel surface there exists in the troposphere a prominent horizontal rotational motion, owing to which the surface of discontinuity in the upper layer, that is, the tropopause, also hangs down (the tropopause funnel) as in the case of a typhoon. Thus in the case of a cold vortex there exists also a warm core in the upper layer just above the cold core.

As a cold vortex usually presents itself as an evident cyclone system in the upper layer, it is also called a high-level cyclone [3] [4]. Conversely to it, the tropical cyclones (typhoons, hurricanes, cyclones and so on) have only a warm core in the central part at least before their maximum stage. They are thus to be called a warm vortex.

We will now discuss theoretically the three-dimensional structure of this perturbation system [5], on which excellent synoptic analyses were done recently by several investigators [3] [6] [7] [14] [18].

In integrating the equations of motion we adopt the following assumptions:

(A) the effect of friction can be considered to be secondary,

(B) the state is axis-symmetrical,

(C) the state is stationary,

(D) the air is autobarotropic.

The meaning of the assumption (A) is that the effect of friction is to be taken into account at places where it is especially large, that is, at the boundary surfaces, because a large wind-velocity jump is expected there irrespective of continuity of wind in an air mass. The assumptions (B), (C) and (D) would be suff.ciently permissible. 
As it was performed in the previous paper "On the Structure or Typhoon" [1], to integrate the equations of motion under the above assumptions, we apply here only the results of the integration.

If we adopt a system of cylindrical coordinates with the axis directed vertically upward from the earth's surface, then it is known from the equation of continuity, that we can apply a stream-line function $\psi$ for the momentum $\left(\frac{v_{r}}{s}, \frac{v_{z}}{s}\right)$. Thus we gat

$$
v_{r}=-\frac{s}{r} \frac{\partial \psi}{\partial z}, \quad v_{z}=\frac{s}{r} \frac{\partial \psi}{\partial r}
$$

Putting these into the equations of motion, we can derive after some devices the following three integrals:

$$
\begin{gathered}
r v+r^{2} \omega \sin \lambda=V(\psi), \\
\frac{s}{r^{2}}\left\{\frac{\partial}{\partial r}\left(s \frac{\partial \psi}{\partial r}\right)+\frac{\partial}{\partial z}\left(s \frac{\partial \psi}{\partial z}\right)\right\}-\frac{s^{2}}{r^{3}} \frac{\partial \psi}{\partial r}+\frac{\left(V-r^{2} \omega \sin \lambda\right) V^{\prime}}{r^{2}}=\Psi^{\prime}(\psi), \\
\frac{1}{2 r^{2}}\left\{\left(s \frac{\partial \psi}{\partial r}\right)^{2}+\left(s \frac{\partial \psi}{\partial z}\right)^{2}+\left(V-r^{2} \omega \sin \lambda\right)^{2}\right\}+g z+\int s d p^{\circ}=\Psi(\psi),
\end{gathered}
$$

where $\lambda$ means the Jatitude, and $v$ is adopted instead of $v_{\theta} . \quad V(\psi)$ and $\Psi(\psi)$ are arbitrary functions of $\psi$ only, and $V^{\prime}$ and $\Psi^{\prime}$ are their derivatives in regard to $\psi$.

$\psi$ expresses a stream-line in the $r z$-plane (meridional plane) containing the cylinder axis. As we are now dealing with a motion symmetrical with respect to the axis, the state of motion is completely known, if only the projection of the state on the meridional plane is found.

$V(\psi)$ and $\Psi(\psi)$ are functions of $\psi$ only, so that they (accordingly $V^{\prime}$ and $\Psi^{\prime}$ also) are constant along a stream-line. Thus, from the equation (2) it is known that the absolute angular momentum around the central axis is conserved along a stream-line in the meridional plane, in such an axis-symmetrical motion system as typhoons and cold vortices (the law of conservation of absolute angular momentum).

Combining (2) with the equation (1), we can know the following: except in the special case when $\psi$ (accordingly $V$ also is everywhere constant in the space, that is, whon absolute angular momentum is everywhere constant, both $v_{r}$ and $v_{z}$ must generally exist in the meridional plane. Thus, the horizontal rotational motion around the central axis is generally accompanied by the convective motion in the meridional plane (meridional circulation), and they can never be separated from each other. Thus, upward and downward motions as well as convergent and divergent ones must generally exist in such axis-symmetrical motion systems as typhoons and cold vortices. This result was derived because we intograted the system of nonlinear partial differential equations of motion, and it would show the existence of cross-stream circulation [3] [19], which is recently attracting special attention in meteorology. The direction of this cross-stream circulation will not be determined from our theory only which assumed autobarotropy. 
It is known further from (2) that the isoplets of the absolute angular momentum around the central axis are the stream-lines of the meridional motion. The wind velocity itself of the horizontal rotational motion varies as

$$
v=V / r-r \omega \sin \lambda \text {, }
$$

so that the wind velocity of the horizontal rotational motion becomes larger as it approaches the central axis, and an astonishingly violent wind will be expected near the center of typhoons and also of some cold vortices.

If we put $r \rightarrow 0$ in (2), then it follows that $v \rightarrow \infty$. Thus we know that in the air mass containing the central axis the absolute angular momentum must be null, that is

$$
v=-r \omega \sin \lambda \text { or } v / r=-\omega \sin \lambda .
$$

This indicates that the air mass forming a core rotates anticyclonically with a constant angular velocity as if it were a solid body. This conclusion holds aslo with respect to the warm, core as well as to the cold one.

(3) is the equation which determines the vorticity component in the meridional plane (the law of conservation of vorticity in a wide sense).

(4) indicates that energy is conserved along a stream-line in the meridional projection (the law of conservation of energy).

\section{Surface of discontinuity}

Now we will consider the conditions at the boundaries and derive relations expressing the shape of the surfaces of discontinuity.

It follows immediately from the kinematical boundary condition, that $\psi=\psi_{0}$ (=const.) at the boundaries (the earth's surface and the internal surfaces of discontinuity). Thus $V$ and $\Psi$ are constants at the boundaries, and absolute angular momentum as well as vorticity (in a wide sense) and energy are conserved along a boundary surface. The stream-lines in the meridional projection must be almost parallel to the boundary surface at least in its vicinity, so that the state of motion is roughly known if only the shape of the surfaces of discontinuity is found. This gives a very favourable standpoint to us who are confronted with the difficult problem of integration.

In order to derive the dynamical boundary cnodition, it will be found that the mere assumption of autobarotropy is insufficient. It is because the value of $\int s d p$, and consequently the form of surface of discontinuity, is different according to the functional form of the specific volume which is dependent on pressure. Therefore we adopt here the wall-known assumption of polytropy as one of the cases of autobarotropy. Then

$$
p s^{n}=p_{0} s_{0}^{n}
$$

and 
(6), $\left(6^{\prime}\right) \quad \int s d p=\frac{1}{\sigma} p^{\frac{n-1}{n}}, \quad$ where $\quad \sigma=\frac{n-1}{n} \frac{q_{0}{ }^{n}}{p_{0}}$.

Therefore we get the following relation from (4), which gives pressura distribution,

$$
p=\left[\sigma \Psi-\sigma g z-\frac{\sigma}{2 r^{20}}\left\{\left(s \frac{\partial \psi}{\partial r}\right)^{2}+\left(s \frac{\partial \psi}{\partial r}\right)^{2}+\left(V-r^{2} \omega \sin \lambda\right)^{2}\right\}\right]^{\frac{n 2}{2 b-1}} .
$$

In the case when there exist two fluid layers, we get from the dynamical boundary condition the following relation, which gives the shape of the surface of discontinuity (suffixes are numbered from the under layer),

$$
\begin{aligned}
& {\left[\sigma_{1} \Psi_{1}-\sigma_{1} g z-\frac{\sigma_{1}}{2 r^{2}}\left\{\left(s \frac{\partial \psi}{\partial r}\right)_{1}^{2}+\left(s \frac{\partial \psi}{\partial z}\right)_{1}^{2}+\left(V_{1}-r^{2} \omega \sin \lambda\right)^{2}\right\}\right]^{\frac{n_{1}}{n_{1}-1}} } \\
= & {\left[\sigma_{2} \Psi_{2}-\sigma_{2} g z-\frac{\sigma_{2}}{2 r^{2}}\left\{\left(s \frac{\partial \psi}{\partial r}\right)_{2}^{2}+\left(s \frac{\partial \psi}{\partial z}\right)_{2}^{2}+\left(V_{2}-r^{2} \omega \sin \lambda\right)^{2}\right\}\right]^{\frac{n_{2}}{n_{2}-1}} . }
\end{aligned}
$$

This relation is a little complex, but can be simplified, if we neglect small terms. First $\left(s \frac{\partial \psi}{\partial r}\right)^{2}=r^{2} v_{z}{ }^{2}$ and $\left(s \frac{\partial \psi}{\partial z}\right)^{2}=r^{2} v_{r}{ }^{2}$ can be neglected as they are of the order of $1 / 100$ of $\left(V-r^{2} \omega \sin \lambda\right)^{2}=r^{2} v^{2}$. Next, as the temperature lapse-rate can be considered to be equal in each air mass, we can put $n_{1}=n_{2}$ (but we must be more careful in treating the stratosphere). Thus we get

$$
z=\frac{\sigma_{1} \Psi_{1}-\sigma_{2} \Psi_{2}+\frac{1}{2}\left(\sigma_{1} V_{1}-\sigma_{2} V_{2}\right) \omega \sin \lambda}{\left(\sigma_{1}-\sigma_{2}\right) g}-\frac{\sigma_{1} V_{1}^{2}-\sigma_{2} V_{2}^{2}}{2\left(\sigma_{1}-\sigma_{2}\right) g} \cdot \frac{1}{r^{2}}-\frac{\omega^{2} \sin ^{2} \lambda}{2 g} \cdot r^{2} .
$$

From this equation, it is known that the effect of the earth's rotation is only effective at a great distance from the center, and that the surface of discontinuity becomes gradually lower, but the effect is sufficiently small and negligible near the center. Further, $\sigma, V$ and $\Psi$ are constant along the surface, therefore, if we put

$$
Z=\frac{\sigma_{1} \Psi_{1}-\sigma_{2} \Psi_{2}}{\left(\sigma_{1}-\sigma_{2}\right) g}
$$

then the shape of the surface of discontinuity near the center is expressed by

$$
z=Z-\frac{\sigma_{1} V_{1}^{2}-\sigma_{2} V_{2}^{2}}{2\left(\sigma_{1}-\sigma_{2}\right) g} \cdot \frac{1}{r^{2}}
$$

in case the effect of the earth's rotation is negligible.

Thus it is known that the surface of discontinuity hangs down infinitely deep or stretches upward infinitely in a funnel shape around the central axis. Hereafter we number the cold core, the surrounding troposphere and the warm core with the suffixes 1, 2 and 3 respectively.

First we consider the surface between the cold core and the surrounding troposphere. As $V_{1}=0$ in the cold core and $\sigma_{1}>\sigma_{2}$,

$$
z=Z_{12}+\frac{\sigma_{2} V_{2}^{2}}{2\left(\sigma_{1}-\sigma_{2}\right) g} \cdot \frac{1}{r^{2}},
$$

where $Z_{12}<0$, because kinetic energy is larger in the surrounding tropospheric air. 
Thus it is known that the surface of the cold front type sustaining the cold core stretches upward infinitely in a funnel shape.

Next wa consider the surface between the warm core and the surrounding troposphere (the tropopause funnel). As $V_{3}=0$ in the warm core and $\sigma_{2}>\sigma_{3}$, the shape of the tropopause funnel is determined by

$$
z=Z_{23}-\frac{\sigma_{2} V_{2}^{2}}{2\left(\sigma_{2}-\sigma_{3}\right) g} \cdot \frac{1}{r^{2}}
$$

where $Z_{23}>0$, because kinetic energy is larger in the surrounding air than in the warm core. Thus it is known that the surface surrounding the warm core (the tropopause funnel) hangs down infinitely deep in a funnel shape.

The surface of discontinuity between the cold and the warm core is expressed thus :

$$
z=Z_{0}-\frac{\omega^{2} \sin ^{2} \lambda}{2 g} \cdot r^{2}
$$

as $V=0$ in both air masses. Thus it is known that the surface between the cold and the warm core is almost horizontal to such an extent that the effect of the earth's rotation can ba neglected.

Thus, between the cold core, the surrounding troposphere and the warm core there exists in the meridional section a triple point, which is a horizontal circle in the three-dimensional space.

As we get from $\left(6^{\prime}\right)$ ( $\Delta$ means the difference between the two sides of the surface of discontinuity),

$$
\frac{\Delta \sigma}{\sigma}=n \frac{\Delta q}{q}=-n \frac{\Delta T}{T}
$$

so the equations (10) and (11) can also be expressed, using the coordinates $\left(r_{0}\right.$, $z_{0}$ ) of the triple point, as

$$
\begin{aligned}
& z=z_{0}-\frac{V^{2}}{2 g n \Delta T_{1}}\left(\frac{1}{r_{0}^{2}}-\frac{1}{r^{2}}\right) \text { at the cold front, } \\
& z=z_{0}+\frac{V^{2}}{2 g n \Delta T_{2}}\left(\frac{1}{r_{0}^{2}}-\frac{1}{r^{2}}\right) \text { at the tropopause funnel. }
\end{aligned}
$$

The inclination of the surface of discontinuity is darived immediately from (9),

$$
\tan \alpha=\frac{d z}{d r}=\frac{\sigma_{1} V_{1}^{2}-\sigma_{2} V_{2}^{2}}{\left(\sigma_{1}-\sigma_{2}\right) g} \cdot \frac{1}{r^{3}}-\frac{\omega^{2} \sin ^{2} \lambda}{g} \cdot r
$$

from which it is known that the inclination of the surface becomes larger as it gets nearer to the central axis. As the wind velocity on one side of the surface is usually much smaller than that on the other side, so we get, neglecting the effect of the earth's rotation,

$$
\tan \alpha=\frac{T}{g n \gamma} \cdot \frac{(\Delta v)^{2}}{\Delta T},
$$

from which it is known that the inclination of the surface of discontinuity is the larger, the larger the wind velocity difference and the smaller the temperature 
difference between the two sides of the surface.

In the axis-symmetrical motion system which has only a warm core in its central part, that is, in tropical cyclones such as typhoons, the core is derived from a descended part, of the stratosphere, and the surface which forms the funnel is a part of the tropopause at least at an advanced stage of the cyclone. It is because of this reason that such a remarkable phenomenon as the eye of typhoon is observed even on the ground surface.

The air forming the warm core is derived from the stratosphere also in the case of cold vortex, but as it is descended from the original stratosphere, so that a temperature lapse-rate almost equal to that in the troposphere is observed in it and an index of polytropy $n$ almost equal to that in the troposphere can be assumed in it:

It would be conceivable in some cases that there can exist a surface of discontinuity between the original stratosphere and the descended warm core (a secondary tropopause). If this surface exists, then its shape would also be expressed approximately by (12) (strictly speaking, it must be deduced from (9), because the temperature lapse-rate is almost null in the original stratosphere). It seems thus that the warm core has a fairly large horizontal extent (radius, about $1000 \mathrm{~km}$ ) at the last stage of typhoons and also of cold vortices. Would it not be this core which E. KLeinschmid [10] calls Körper after his synoptic analysis? As is mentioned above, the warm core has, as an isolated air mass, an independent internal motion from the surrounding air and its vertical extent is fairly reduced from under by the effect of friction as will be stated later.

One might interpose an objection to our regarding the tropopause as an ordinary internal surface of discontinuity [11]. It would, however, be sure that the state of discontinuity at the tropopause is a natural phenomenon which is to be explained from more fundamental physical principles, and is not a fundamental principle itself by which one is always to explain the dynamical phenomena of the atmosphere. The equations which describe the dynamics of the atmosphere are composed of a system of simultaneous partial differential equations of the first order, if we put the effect of friction out of consideration. Thus it sjems to us that the botindary conditions are sufficient if they are given by the relations concerning the dependent variables themselves, which are called the kinematical and the dynamical conditions applied to our theory.

Only in the case when friction is effective, the differential equations of motion become of the second order, and in this case it would be necessary to give relations of their derivatives as boundary conditions.

As it is, howaver, quite hopaless, for the present, to try to integrate strictly the equations of motion of frictional fluid, we will take the effect of friction into consideration as a secondary factor. Then the tropopause becomes a surface of the first order [1], as will ba seen in our next article. 


\section{The effect of friction}

Usually we assume that the dependent variables are continuous in an air mass in treating the motion of the atmosphere, and therefore the effect of friction is not so large in an air mass. But the conditions are quite different at the boundaries (at the earth's surface and at the internal surfaces of discontinuity), where the dependent variables may change their values abruptly. For instance in developed typhoons, it is considered that there exists a jump of wind velocity of $100 \mathrm{~m} / \mathrm{s}$ or more at the tropopause funnel between the warm core and the surrounding troposphere. Almost the same can be said of the case of cold vortex. In those cases we can never neglect the effect of friction (the external friction). Hereafter we will consider only this effect of external friction at the surface of discontinuity.

Mathematically it is not easy to treat the effect of friction even as a secondary factor. But intuitively this effect is quite clear. That is, wind velocity weakens on one side of the surface where it was initially larger, and strengthens an the other side where it was initially smaller, and thus the wind velocity itself changes gradually from one side to the other without discontinuity. In a word, it is that the motion is smoothed through the mixing of air. In order to estimate the width of the friction layer (the mixing layer) generated at the surface of discontinuity, we applied previously the conception of shearing instability [1] [11] [12]. Of course it would not be the only way of explanation, but it seems to us that the result derived is fairly satisfactory. Therefore it will be applied also to the case of cold vortex.

By this method, the width $L$ of the mixing layer generated at the surface of discontinuity is expressed by

$$
L=\frac{2 \pi T}{g} \cdot \frac{(\Delta v)^{2}}{\Delta T}\left\{1-\frac{1}{2}\left(\frac{T}{g n r}\right)^{2} \cdot\left(\frac{(\Delta v)^{2}}{\Delta T}\right)^{2}\right\},
$$

where the magnitude of the second term in the bracket on the right hand side is sufficiently small as compared with 1 and is usually negligible. Thus it follows that the width of the mixing layer is the larger, the larger the wind velocity difference and the smaller the tempereatur difference between the two sides of the surface of discontinuity. Comparing this result with that derived from $\left(14^{\prime}\right)$, it follows further that the width of the mixing layer is the larger at places where the inclination of the surface is the larger. Thus it is seen that both the inclination of the surface and the width of the mixing layer ara largest at the lower part of the tropopause funnel.

Owing to the formation of this friction layer, the surface of discontinuity becomes diffuse, and there hardly exists in the atmosphere such pure surfaces as are considered in frictionless motion. It seems to be only where frontogensis, especially dus to the effect of rain-fall, is most effective, that this layer is sufficiently small in width and can be regarded as a pure surface of discontinuity. At the surface 
of discontinuity which remains for a long time in the atmosphere, the effect of friction will necessarily appear and the surface will change itself into a friction layer (transition layer). Thus, if one wants to analyse a front dynamically as well as thermo-dynamically from aerological data, then one must direct his attention to the point where temperature lapse-rate changes abruptly, as is shown later in Fig. 2 . Not only at the earth's surface, but also in the free atmosphere, there exist places where the effect of friction predominantly appears, and those are the places where the surface of discontinuity of the frictionless motion ought to exist. The point where temperature lapse-rate changes abruptly is the boundary between the domain where the effect of friction is predominant and that where it is negligible.

The surface of discontinuity between the troposphere and the stratosphere would have always to exist if the effect of friction be negligible, therefore one must consider that the effect of friction is especially predominant at the tropopause. It seems to us that the reason why the tropopause appears as a surface of discontinuity of the first order lies in the above-mentioned circumstance. However, not only the tropopause but almost all the surfaces of discontinuity also appear actually as surfaces of the first order. One is inclined to consider the tropopause only as the first order, because of the reason that there exists usually no temperature decrease in the stratosphere.

\section{Three-dimensional structure of the isolated cold vortex}

From the above considerations we know the three-dimensional structure of the axis-symmetrical cold vortex. In a cold vortex, owing to the centrifugal force due to a prominent rotational motion of the surrounding tropospheric air, a cold air mass is solitarily sustained in the lower layer containing the central axis, and a warm air mass is drawn down in the upper layer from the stratosphere. The motion in the cold and the warm cores is expressed by the equation ( $\left.2^{\prime \prime}\right)$, that it, the cores rotate slowly and anticyclonically with a constant angular velocity. The shape of the surface of discontinuity between the cores is expressed by (12) and is almost horizontal. The surface surrounding the cold core is expressed by the equation (10), and it is the cold front in the case when the vortex is generated by a cut-off process of polar air [3] [7] [13] [14]. Thus the cold core takes a form of a cut-off funnel, but the surfaces bordering it are diffused owing to the effect of friction.

The warm core lying above the cold one is surrounded by the tropopause funnel which is expressed by the equation (11). In some cases a secondary tropopause which may be expressed by (12) will be found between this core and the original stratosphere. It is considered that the effect of friction is the most predominant in the motion of the stratosphere, so that the integrals which were derived from the assumption of frictionless motion will not probably be applicable there. 
The surrounding (tropical) tropospheric air rotates cyclonically with a prominent speed around the funnel-shaped surfaces and the main part of the motion is expressed by the equation (2). In this air mass, however, there exists together with this rotational horizontal motion a convective one, that is, a meridional circulation which is expressed by the equation (3).

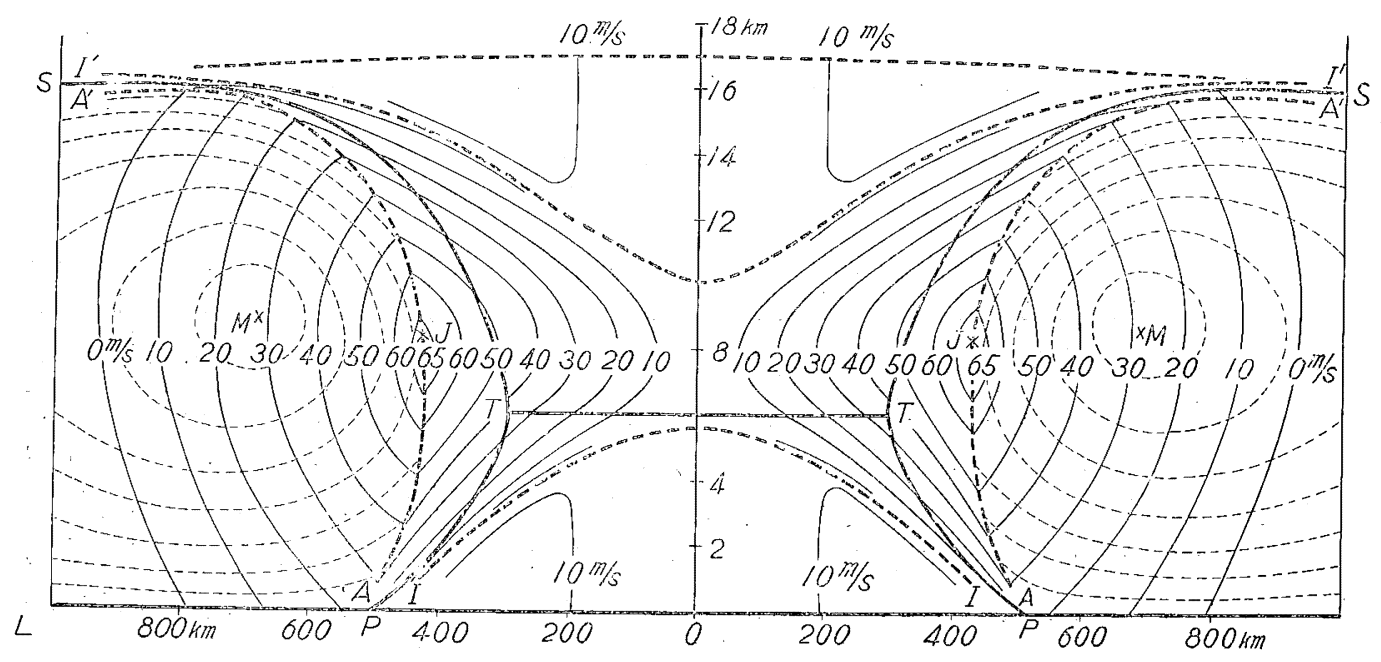

Fig. 1. Vertical Section of an Axis-symmetrical Cold Vortex

Heavy full lines indicate the original surfaces of discontinuity; TP: frontal surface within the troposphere, TS: tropopause funnel, TT: tropopause between the warm and the cold core. Heavy broken lines indicate the side-surfaces of the mixing layer and the secondary tropopause (if possible), thin full lines isovels of the circulatory horizontal wind, and thin broken lines stream-lines of the convective meridional motion. $\mathrm{T}$ : triple poin $\mathrm{\imath}, \mathrm{J}$ : center of the jet stream.

The stream-lines in the meridional plane, which are the projection of the threedimensional motion upon that plane, are almost parallel to the boundary. As the shapes of the surfaces of discontinuity are now determined, the state of motion in this tropical air mass is sufficiently known. If we assume the direction of the meridional circulation to be convergent in the lower layer and divergent in the upper layer as is usually the case, then the above discussion shows that the velocity of the ascending current is far larger than that of the descending current. Thus the three-dimensional motion of an air particle in the tropical air mass is such that it moves spirally along a surface of revolution, the vertical section of which is represented $d_{\overline{\mathrm{A}}}^{\text {by }}$ a stream-line in the meridional plane.

As this convective motion transports the momentum of horizontal rotational motion, so that wind velocity becomes increasingly larger as it gets nearer to the central axis, as is shown by $\left(2^{\prime}\right)$. Thus, if the effect of friction be put out of consideration, then the maximum of wind velocity, that is, a jet stream would be found at the triple point. Actually the wind velocity is weakened through friction. The 
effect of friction is especially predominant at the tropopause funnel, therefore the center of the jet straam turns away from the triple point and its strength is weakened.

In the case when the mixing layer reaches to the central axis, the wind velocity distribution in the layer is expressed approximately by the constancy of the vertical vorticity component

$$
v / r=2 c .
$$

That is, the air in the mixing layer rotates cyclonically with a constant angular velocity, when the layer reaches to the central axis, but the angular velocity generally depends upon the height of the rotating layer. Thus, at the heights where the mixing layer reaches to the central axis, the state of motion in a horizontal plane is expressed approximately by RANKINE's combined vortex (a high-level cyclone) [3] [4] [7]. That is, the wind velocity along a horizontal line increases straightly from mull at the center to a maximum (the jet stream) at the outer side of the mixing layer, and then decreases almost in inverse proportion to the central distance, in the case when the mixing layer reaches to the axis. This distribution of wind corresponds wall to the horizontal distribution of pressure which will be stated later.

In the case when the mixing layer does not reach to the central axis, there exists a weak, anticyclonic wind domain near the center of core, and from the inner side of the mixing layer the wind velocity increases straightly up to its maximum at the outer side of the layer, and then it decreases almost in inverse proportion to the central distance. As the anticyclonic wind in the cores is very weak, it would be difficult to determine it from a synoptic analysis. Roughly analysed, a cold vortex will appear thus as a cyclone at all heights, but it must be noted that the height interval where the vortex is really a cyclone is limited to the uppermost part of the troposphere and to the lowermost part of the stratosphere, where the effect of friction is the most predominant.

The horizontal distribution of pressure is expressed by (7) in the tropical troposphere. In the mixing layer, it is derived from (16) concerning wind; in the core, from (7).

It is seen, thus, that pressure is weakly anticyclonic in the core and cyclonic in the mixing layer and also in the surrounding troposphere, and that the axis becomes the center of low pressure at the heights where the mixing layer reaches to the central axis (high-level cyclone), but in case the core is not eroded completely, there remains a circular low pressure bəlt around the cantral axis. Pressure gradient is largest at the outer side of the mixing layer and corresponds to the existencs of the jet stream. In the tropical troposphere, pressure becomes rather lower in the part very far from the axis (see (7)).

As air is autobarotropic in our treatment, the distribution of temperature coincides with that of pressura except in the mixing layer, where temperature changes without 
discontinuity from one air mass to another (See Fig. 2). Thus, temperature decreases in the tropical troposphere up to the outer side of the mixing layer, from where it increases (or decreases) straightly in the mixing layer and from the inner side of the layer it increases very slowly up to its maximum at the center of warm (or cold) core. Thus, temperature minimum in the upper layer exists at the outer side of the mixing layer and in the lower layer at the inner side of the mixing layer.
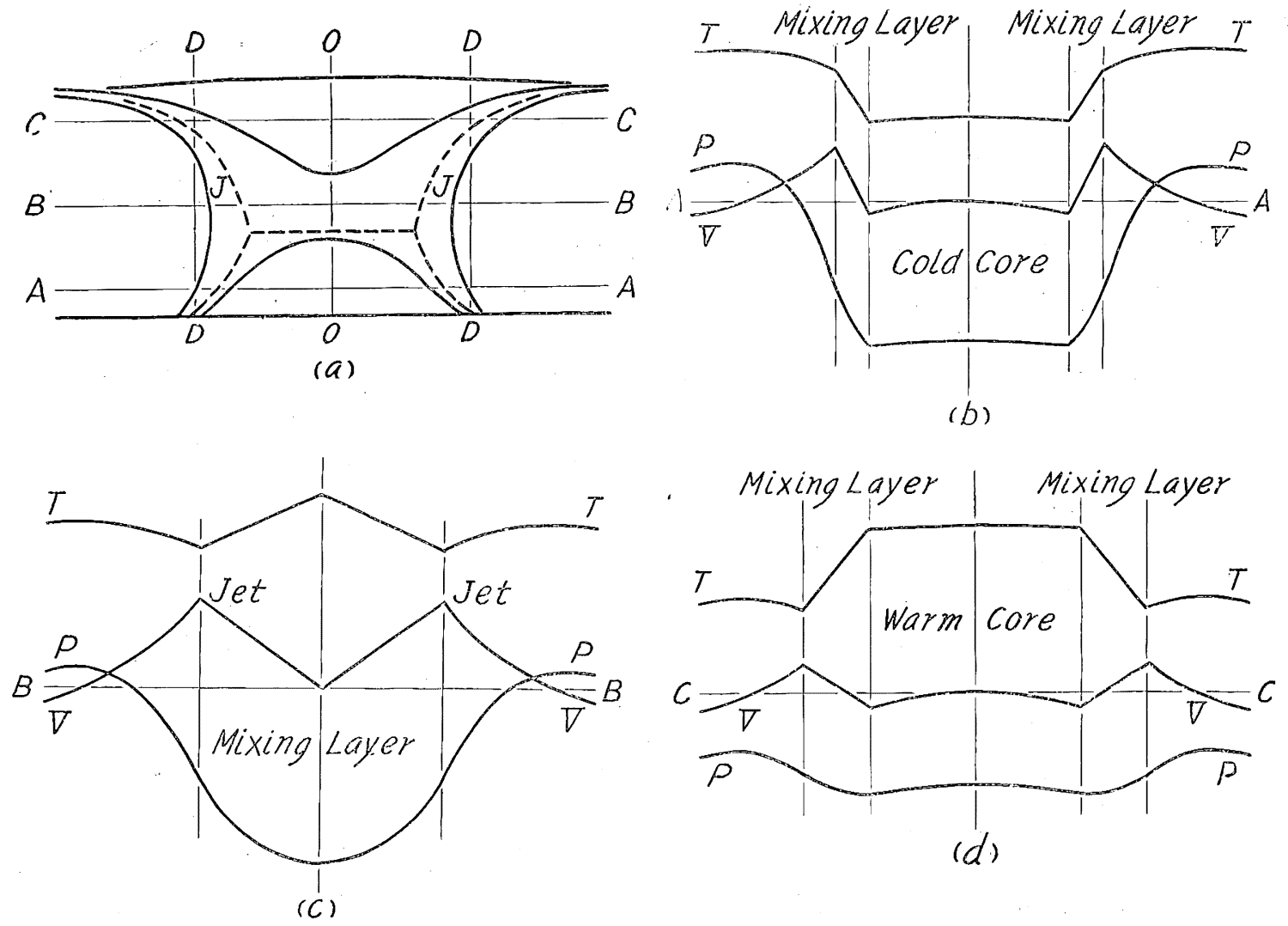

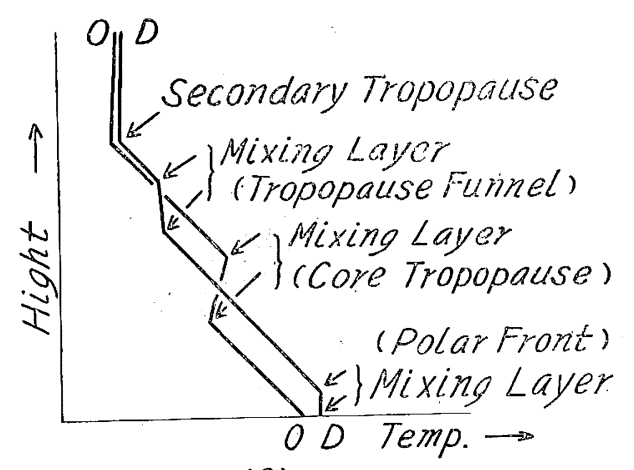

(e)
Fig. 2. Distribution" of Meteorological Elements in a Cold Vortex.

(a) indicates the positions of horizontal and vertical lines along which the distribution of meteorological elements is shown as (b), (c), (d) and (e). (b), (c) and (d) show the distribution of temperature $(\mathrm{T})$, bressure $(\mathrm{P})$ and wind velocity $(\mathrm{V})$ along the horizontal lines $\mathrm{AA}, \mathrm{BB}$ and $\mathrm{CC}$ in (a) respectively. (e) shows the vericical distribution of temperature along $\mathrm{OO}$ and $\mathrm{DD}$ in (a). 
The distribution of meteorological elements thus derived is shown in Fig. 2. In preparing Fig. 1 we adopted the following values:

$\lambda=45^{\circ}, T_{0}=280^{\circ}$ (mean value at the ground), $r_{0}=300 \mathrm{~km}, z_{0}=6 \mathrm{~km}$,

$\Delta T_{1}=16^{\circ} \mathrm{C}, \Delta T_{2}=12^{\circ} \mathrm{C}, V=3.30 \times 10^{11} \mathrm{CGS}$ (corresponding to $v=0$ at the boundary $800 \mathrm{~km}$ distant from the center),

where the values of $\Delta T$ would seem to be a little too large, but judging from the analyses after E. PALMÉN etc. [3] [7] [8], it seems to us that such temperature differences may frequently appear at the front.

Applying these values, we calculated the surface of discontinuity from the equations (10a) and (11a), the wind velocity from $\left(2^{\prime}\right)$ and the thickness of the mixing layer from (15), and drew Fig. 1. In Fig. 1 the stream-lines in the tropical troposphere were drawn inferred from the shape of the boundary and considering the change of specific volume with height. The value of $V$ is assumed to increase a little toward the point $M$ where $\psi$ takes its extremum value (the change of $V$ due to $\psi$ is usually very small and the value of $V$ is very near to a constant).

As is seen from Fig. 1, a cold vortex is composed for the most part of a wind system which rotates cyclonically around the central axis with a considerable speed, except the parts rotating slowly and anticyclonically around the axis in the lower and the upper layers.

When the cold core is large in extent, there appears a clear anticyclone on the earth's surface. In such a case, our theory gives the dynamical structure of anticyclone. When the cold core is small and completely eroded through friction, there appears a cyclone on the earth's surface as well. We refer to a description in E. PALMÉN's paper [3] on this point.

In Fig. 2 the distribution of meteorological elements is shown along several horizontal or vertical lines in the meridional section. It is seen from Fig. 2 that surfaces of discontinuity in a strict sense do not appear in the atmosphere and are transformed into transition layers (friction layers) owing to the effect of friction. Not only the tropopause, but almost all the surfaces of discontinuity thus appear actually as surfaces of the first order. It would, however, be usually difficult to find out the side-surface of the transition layer in the stratosphere because of the predominance of friction.

In our treatment, the air. is assumed to be autobarotropic, but actually in the atmosphere the effect of water will appear predominantly at some places. Thus, for instance, frontogenesis may sometimes be observed at the front near the gronud surface, and the front would partly present itself as a surface of the zeroth order. Further, when water content is large in the surrounding tropospheric air, then, in the mixing layer, where the wet air mixes with the dry air of the warm core, temperature will be reduced considerably lower than that shown in Fig. 2 owing to 
the effect of mixing. We did not touch upon these effects hitherto, because it is impossible to treat them all within a single theory.

\section{Additional notes}

As is seen in the figures, the vertical section of an axis-symmetrical cold vortex is very similar to that of the circumpolar vortex [11] [15] [16] [17]. In the circumpolar vortex, it is known that the tropopause descends remarkably to the north of the jet stream, and that the cold front is found under the jet stream. If we regard the polar troposphere as a large cold core, then it will be found that the circumpolar vortex is nothing but a large cold vortex.

To the south of the polar front, there are observed frequently cut-off cold vortices. These are nothing but children, which have the same characteristics as their mother, the large circumpolar vortex.

We discussed previously the structure of the typhoon, which is to be called a warm vortex. It will be found that the vertical section of a typhoon is also very similar to that of a cold vortex. The only difference is the absence of a cold core.

Our theory shows further the dynamical structure of an anticyclone.

The pronounced similarity between these perturbatior systems is caused by the fact that they are based upon the same fundamental principles, that is, the three conservation laws expressed by the equations (2), (3) and (4).

\section{References}

[1] SAto, T., 1951: On the Structure of Typhoon, yet unpublished.

[2] Scherhac, R., 1948: Neue Methoden der Wetteranalyse und Wetterprognose.

[3] Palmín, E, 1949: On the Origin and Structure of High-Level Cyclones South of the Maximum Westerlies, Tellus, 1, No. 1.

[4] RIEHL, H., 1950: Variations in the Structure of High-Level Cyclones, Bulletin of American Meteorological Society, 31, No. 8.

[5] Bjerknes, V., 1921: On the Dynamics of the Circular Vortex with Applications to the Atmosphere and Atmospheric Vortex and Wave Motions, Geofysiske Publikasjoner 2, No. 4.

[6] Crocker, A.M., W.L. Gonson and C.M. Penner, 1947: Frontal Contour Charts, Journal of Meteorology, 4, No. 3.

[7] Hsien, Y.P., 1949: An Investigation of a Selected Cold Vertex over North America, Jour nal of Meteorology, 6, No. 6.

[8] PALmḱn, E. and C.W. Newron, 1951: On the Three-Dimensional Motions in an Outbreak of Polar Air, Journal of Meteorology, 8, No. 1.

[9] Rrehl, H., 1950: A Model of Hurricane Formation, Journal of Applied Physics, 21, Sept.

[10] Klfinschmidr, E., 1950: Ueber Aufbau und Entstehung von Zyklonen, Met. Rundsch., 3. Jahrg., Heft 1/2-3/4. 
[11] SAto, T., 1951: Dynamics of the Jet Stream, Papers in Meteorology and Geophysics, 2, No. 2.

[12] Bjerknes, V. and his Collaborators, 1933: Physikalische Hydrodynamik.

[13] PALMÉn, E. and K.M. NAGLER, 1949: The Formation and Structure of a Large-Scale Disturbance in the Westerlies, Journal of Meteorology, 6, No. 4.

[14] Hsirn, Y.P., 1950: On the Formation of Shear Lines in the Upper Atmosphere, Journal of Meteorology, 7, No. 6.

[15] Staff Members Of The Departmenar Of Meteorology, University Of Cificago, 1942: On the General Circulation of the Atmosphere in Middle Latitudes, Bulletin of American Meteorological Society, 28, No. 6.

[16] PALmín, E., 1948: On the Distribution of Temperature and Wind in the Upper Wester. lies, Journal of Meteorology, 5, No. 1.

[17] Nyвеrg, A., 1949: An Aerological Study of Large-Scale Atmospheric Disturbances, Tellus, 1 , No. 1 . 